\title{
A numerical study of divergence-free kernel approximations
}

\author{
Arthur A. Mitrano, Rodrigo B. Platte \\ School of Mathematical and Statistical Sciences \\ Arizona State University, Tempe, USA
}

\begin{abstract}
Approximation properties of divergence-free vector fields by global and local solenoidal bases are studied. A comparison between interpolants generated with radial kernels and multivariate polynomials is presented. Numerical results show higher rates of convergence for derivatives of the vector field being approximated in directions enforced by the divergence operator when a rectangular grid is used. We also compute the growth of Lebesgue constants for uniform and clustered nodes and study the flat limit of divergence-free interpolants based on radial kernels. Numerical results are presented for two and three dimensional vector fields.
\end{abstract}

Keywords: Radial basis functions, divergence-free, finite-differences, spectral methods

\section{Introduction}

In physical applications, such as fluid mechanics, meteorology, and electromagnetics, vectorial data needs to be interpolated on a given set of nodes. This can be done by interpolating each component of the vector field independently. However, when the field is divergence-free or curl-free, more accurate approximations might be obtained if the connection between the components of the vector field is taken into consideration.

The present work is motivated by incompressible fluid flow simulations with constant density, where the velocity field is divergence-free as a consequence of mass conservation. It is well known (see [16] for instance) that instability can arise in incompressible fluid flow simulations if the divergence-free condition is not met. When approximations are not divergence-free, projection methods applied at each time-step can be used to enforce numerical mass conservation, see [19]. An alternative to those projection methods is to use a divergence-free basis to approximate such vector fields.

Divergence-free radial kernels where explored in [17, 1, 18, 2, 3, using a variational spline setting. In 1994, matrix-valued radial basis functions (RBFs) were introduced to approximate generalized interpolation problems on scattered data [26. In that framework, the divergence-free kernels are generated using linear side conditions. Stability estimates for those interpolants were given in [26, 24] and improved in [15], while error estimates for functions on native spaces were derived in [23] and later extended for rougher functions in [13, where Sobolev-type error estimates were derived. Similar divergence-free interpolants were developed in [27], where the interpolant fits a vector field of zero divergence that is tangent to an orientable surface. Error and stability estimates for those interpolants on the sphere were presented in [14].

In this paper, we implement global and local divergence-free approximations based on the radial kernels presented in [27] and multivariate polynomials. Of particular interest is the accuracy of the derivatives of these interpolants when restricted to planar regions. We also present a numerical investigation of the flat limit $(\varepsilon \rightarrow 0)$ of divergence-free radial kernel interpolants. For the three-dimensional case, we explore the accuracy of the kernels proposed in $[26$.

Email addresses: amitrano@asu.edu (Arthur A. Mitrano), rbp@asu.edu (Rodrigo B. Platte) 

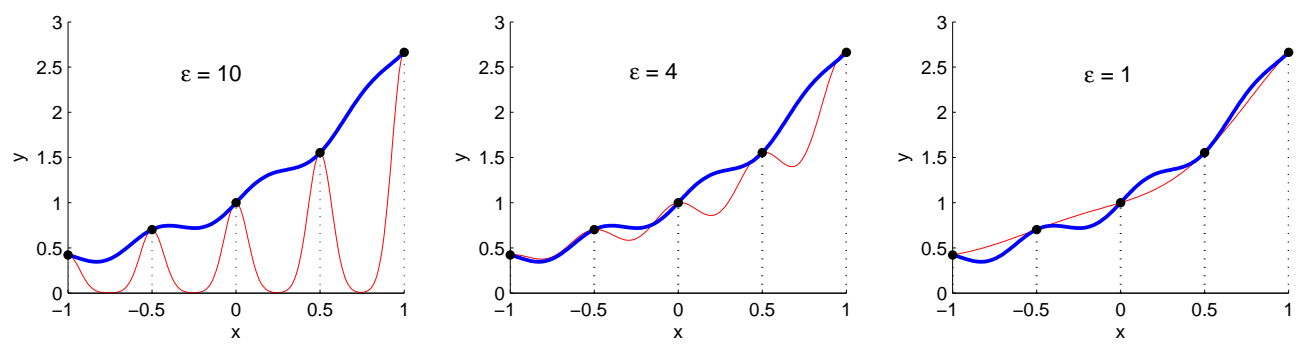

Figure 1: Gaussian RBF interpolation of $f(x)=\exp (x)+0.1 \sin (10 x)$ using three values of $\varepsilon$.

\section{Background concepts}

\subsection{Standard RBF interpolation and differentiation matrices}

Given a function function $f$, we define its radial basis interpolant as

$$
s_{f, N}(\boldsymbol{x})=\sum_{k=1}^{N} c_{k} \phi\left(\varepsilon\left\|\boldsymbol{x}-\boldsymbol{x}_{k}\right\|\right),
$$

where $\phi$ is the basic function (or kernel) and the coefficients $c_{k}$ are calculated such that $s_{f, N}\left(\boldsymbol{x}_{j}\right)=f\left(\boldsymbol{x}_{j}\right)$ for $k=1, \ldots, N$. The interpolation points are called centers and $\varepsilon$ the shape parameter. Enforcing the interpolation condition leads to the linear system

$$
\left[\begin{array}{cccc}
\phi\left(\varepsilon\left\|\boldsymbol{x}_{1}-\boldsymbol{x}_{1}\right\|\right) & \phi\left(\varepsilon\left\|\boldsymbol{x}_{1}-\boldsymbol{x}_{2}\right\|\right) & \ldots & \phi\left(\varepsilon\left\|\boldsymbol{x}_{1}-\boldsymbol{x}_{N}\right\|\right) \\
\phi\left(\varepsilon\left\|\boldsymbol{x}_{2}-\boldsymbol{x}_{1}\right\|\right) & \phi\left(\varepsilon\left\|\boldsymbol{x}_{2}-\boldsymbol{x}_{2}\right\|\right) & \ldots & \phi\left(\varepsilon\left\|\boldsymbol{x}_{2}-\boldsymbol{x}_{N}\right\|\right) \\
\vdots & \vdots & \ddots & \vdots \\
\phi\left(\varepsilon\left\|\boldsymbol{x}_{N}-\boldsymbol{x}_{1}\right\|\right) & \phi\left(\varepsilon\left\|\boldsymbol{x}_{N}-\boldsymbol{x}_{2}\right\|\right) & \ldots & \phi\left(\varepsilon\left\|\boldsymbol{x}_{N}-\boldsymbol{x}_{N}\right\|\right)
\end{array}\right]\left[\begin{array}{c}
c_{1} \\
c_{2} \\
\vdots \\
c_{N}
\end{array}\right]=\left[\begin{array}{c}
f\left(\boldsymbol{x}_{1}\right) \\
f\left(\boldsymbol{x}_{2}\right) \\
\vdots \\
f\left(\boldsymbol{x}_{N}\right)
\end{array}\right] .
$$

Some examples of radial kernels (basic functions) are:

$$
\begin{aligned}
& \phi(r)=\exp \left(-r^{2}\right) \\
& \phi(r)=1 /\left(1+r^{2}\right)^{\beta} \\
& \phi(r)=\sqrt{1+r^{2}}
\end{aligned}
$$

\section{Gaussians}

generalized inverse multiquadrics

multiquadrics

The convergence properties of an RBF expansion depend on the smoothness of $\phi$ and the node distribution. The functions in the list above are all analytic and lead to approximations that converge exponentially when the target function is sufficiently smooth 28 .

The shape parameter $\varepsilon$ plays a key role in the accuracy of the approximations and conditioning of interpolation matrices. Figure 1 shows the role of $\varepsilon$ and how it changes the interpolant shape. A larger value of $\varepsilon$ leads to a more localized interpolant, and a smaller value gives a global approximation. In the limit $\varepsilon \rightarrow 0$, it was shown in [4], for the univariate case, and in [21, 22, 32, for multivariate interpolation, that smooth RBF interpolants converge to polynomial interpolants on the same nodes.

Basis functions of finite smoothness are also popular. They include linear splines, $\phi(r)=r$, cubic splines $\phi(r)=r^{3}$ and compactly-supported radial functions, also known as Wendland's functions [35. Due to singularities in the RBF expansion (jump in derivatives), these functions lead to approximations that converge algebraically, with the order depending on the number of smooth derivatives. For simplicity, in this article we focus only on Gaussians and inverse multiquadrics.

Differentiation matrices can be obtained from (1), which are linear maps from function values at the nodes $x_{k}$ into derivative values of (11) at the same nodes. These matrices can then be used to solve differential equations, as is common practice in spectral (collocation) methods. 
To find a differentiation matrix $D_{x}$, we differentiate (1) with respect to $x$,

$$
\frac{d}{d x} s_{f, N}(\boldsymbol{x})=\sum_{k=1}^{N} c_{k} \frac{d}{d x} \phi\left(\varepsilon\left\|\boldsymbol{x}-\boldsymbol{x}_{k}\right\|\right)
$$

Evaluating this last expression at the collocation points $\boldsymbol{x}_{i}$ we get

$$
\boldsymbol{f}^{\prime}=A_{x} \boldsymbol{c}
$$

where the entries of the matrix $A_{x}$ are $\left.\frac{d}{d x} \phi\left(\varepsilon\left\|\boldsymbol{x}-\boldsymbol{x}_{k}\right\|\right)\right|_{\boldsymbol{x}=\boldsymbol{x}_{i}}$.

To obtain $D_{x}$, we use (2) to write $\boldsymbol{c}=A^{-1} \boldsymbol{f}$. Then (3) becomes

$$
\boldsymbol{f}^{\prime}=A_{x} A^{-1} \boldsymbol{f}
$$

so that the differentiation matrix is given by

$$
D_{x}=A_{x} A^{-1} .
$$

\subsection{Divergence-free $R B F$ interpolation}

In [27, a method for fitting divergence-free vector fields tangent to a two-dimensional surface was presented. In this article we use the kernels presented in 27 restricted to a planar region in $\mathbb{R}^{2}$.

Let $\boldsymbol{t}_{1}, \ldots, \boldsymbol{t}_{N}$ be samples of a vector field at the points $\boldsymbol{x}_{1}, \ldots, \boldsymbol{x}_{N}$, then, according to [27], a divergence-free interpolant is given by

$$
\boldsymbol{t}(\boldsymbol{x})=\sum_{k=1}^{N} \Psi\left(\boldsymbol{x}, \boldsymbol{x}_{k}\right) \boldsymbol{s}_{k}
$$

where $s_{k}$ are coefficient vectors to be calculated such that (4) interpolates the data and

$$
\Psi(\boldsymbol{x}, \boldsymbol{y})=F(r)\left(\boldsymbol{n}_{\boldsymbol{y}} \boldsymbol{n}_{\boldsymbol{x}}^{T}-\boldsymbol{n}_{\boldsymbol{y}}^{\boldsymbol{T}} \boldsymbol{n}_{\boldsymbol{x}} I\right)-G(r)\left(\boldsymbol{n}_{\boldsymbol{x}} \times(\boldsymbol{x}-\boldsymbol{y})\right)\left(\boldsymbol{n}_{\boldsymbol{y}} \times(\boldsymbol{x}-\boldsymbol{y})\right)^{T},
$$

with

$$
F(r)=\frac{1}{r} \phi^{\prime}(r), \quad G(r)=\frac{1}{r}\left(\frac{1}{r} \phi^{\prime}(r)\right)^{\prime}=\frac{1}{r} F^{\prime}(r)
$$

and $\boldsymbol{n}_{\boldsymbol{v}}$ denoting the normal vector of the surface at the point $\boldsymbol{v}$.

Considering the case where the surface is a plane perpendicular to the $z$-axis, and that the vector field has no component in the $z$ direction, (5) can be written as

$$
\Psi(\boldsymbol{x}, \boldsymbol{y})=-F(r)\left[\begin{array}{ll}
1 & 0 \\
0 & 1
\end{array}\right]-G(r)\left[\begin{array}{cc}
\left(x_{2}-y_{2}\right)^{2} & -\left(x_{1}-y_{1}\right)\left(x_{2}-y_{2}\right) \\
-\left(x_{1}-y_{1}\right)\left(x_{2}-y_{2}\right) & \left(x_{1}-y_{1}\right)^{2}
\end{array}\right] .
$$

To find the coefficient vectors $\boldsymbol{s}_{k}$, we evaluate the interpolant at the collocation points $\boldsymbol{x}_{j}$

$$
\boldsymbol{t}_{j}=\sum_{k=1}^{N} \Psi\left(\boldsymbol{x}_{j}, \boldsymbol{x}_{k}\right) \boldsymbol{s}_{k} \quad j=1, \ldots, N .
$$

Letting $\boldsymbol{t}_{j}=\left(u_{j}, v_{j}\right)$ and $\boldsymbol{s}_{k}=\left(s_{k}^{u}, s_{k}^{v}\right)$, we can rewrite [6] as

$$
\boldsymbol{d}=A \boldsymbol{c},
$$

with $\boldsymbol{d}=\left[u_{1}, v_{1}, \cdots u_{N}, v_{N}\right]^{T}, \boldsymbol{c}=\left[s_{1}^{u}, s_{1}^{v}, \cdots s_{N}^{u}, s_{N}^{v}\right]^{T}$ and $A$ the $2 N \times 2 N$ matrix composed of the blocks $\Psi\left(\boldsymbol{x}_{j}, \boldsymbol{x}_{k}\right)$. 
Letting $\Psi_{1}$ and $\Psi_{2}$ denote the first and second columns of $\Psi\left(\boldsymbol{x}, \boldsymbol{x}_{k}\right)$, respectively,

$$
\begin{aligned}
\nabla \cdot \boldsymbol{t}(\boldsymbol{x}) & =\sum_{k=1}^{N} \nabla \cdot\left(\Psi\left(\boldsymbol{x}, \boldsymbol{x}_{k}\right) s_{k}\right) \\
& =\sum_{k=1}^{N}\left[s_{k}^{u} \nabla \cdot \Psi_{1}+s_{k}^{v} \nabla \cdot \Psi_{2}\right]=0,
\end{aligned}
$$

since a direct calculation shows that $\nabla \cdot \Psi_{1}=0=\nabla \cdot \Psi_{2}$.

The existence of a solution to the linear system (7) depends on the invertibility of the matrix $A$. For positive definite kernels $\phi$, it was shown in [27] that this matrix is positive-definite, therefore invertible.

Differentiation matrices based on divergence-free kernels can be computed by repeating the steps presented in Section 2.1 and we omit the details.

\subsection{Divergence-free polynomial interpolation}

For reference, we also explore approximations by polynomial vector fields. To this end, consider a polynomial stream function of degree $n$ given by

$$
\psi(x, y)=\sum_{i=0}^{n} \sum_{j=0}^{n} a_{i j} x^{i} y^{j}, \quad n \in \mathbb{N} .
$$

If the components of the vector field are given by $\boldsymbol{p}=\left(p_{1}, p_{2}, 0\right)=\nabla \times(0,0, \psi)=\left(\psi_{y},-\psi_{x}, 0\right)$, then $\nabla \cdot \boldsymbol{p}=0$. To find the coefficients $a_{i j}$ we use the condition $\boldsymbol{p}\left(\boldsymbol{x}_{k}\right)=\boldsymbol{f}\left(\boldsymbol{x}_{k}\right)$ for each interpolation point $\boldsymbol{x}_{k}$, where $f$ is the target vector field. Depending on the degree $n$ of the stream function, the linear system for the coefficients $a_{i j}$ may or may not have a solution. In the latter case, we use the solution in the least square sense, and in case of multiple solutions, we use a basic solution (as computed by Matlab). Notice that we can take $a_{00}=0$ since the velocity field does not depend on the constant term. We discuss the relationship between the polynomial degree and the number of data points in Section 3.2 .

\subsection{Divergence-free finite differences}

Both RBF and polynomial divergence-free expansions can be used as a basis for finite difference approximations. RBF based finite differences have been used in [33, 36, 8, 6] for scalar functions. Divergence-free finite difference weights can be found using an analogous procedure to the one used to generate standard finite difference formulas. More specifically, in the case of RBFs, we solve the linear system (7) using a local stencil and evaluate the derivatives of (4) at the stencil center point. Since partial derivatives are translation invariant, we can consider our stencil centered at the origin. Global approximations are often more accurate than local ones, but computational cost and conditioning make them impractical for many applications. This article addresses both the global and local approximation schemes.

\section{Numerical results}

\subsection{A numerical example}

The objective of this section is to explore the methods presented above through numerical experiments. Our test function is given by

$$
\boldsymbol{f}(x, y)=\left(\frac{\sin \left(k_{1}(x-0.1)\right) \cos \left(k_{2}(y-0.2)\right)}{k_{1}},-\frac{\cos \left(k_{1}(x-0.1)\right) \sin \left(k_{2}(y-0.2)\right)}{k_{2}}\right), \quad(x, y) \in \mathbb{R}^{2},
$$

which has $\nabla \cdot \boldsymbol{f}=0$ for all $k_{1}, k_{2} \in \mathbb{R} \backslash\{0\}$.

In our numerical experiments we use $k_{1}=7$ and $k_{2}=7$. Figure 2 illustrates the vector field and its divergence-free interpolant computed using (4). The radial kernel used is a Gaussian with shape parameter $\varepsilon=2$. There are $N=256$ data sites distributed equally on both dimensions of the unit square $[-1,1]^{2}$. 


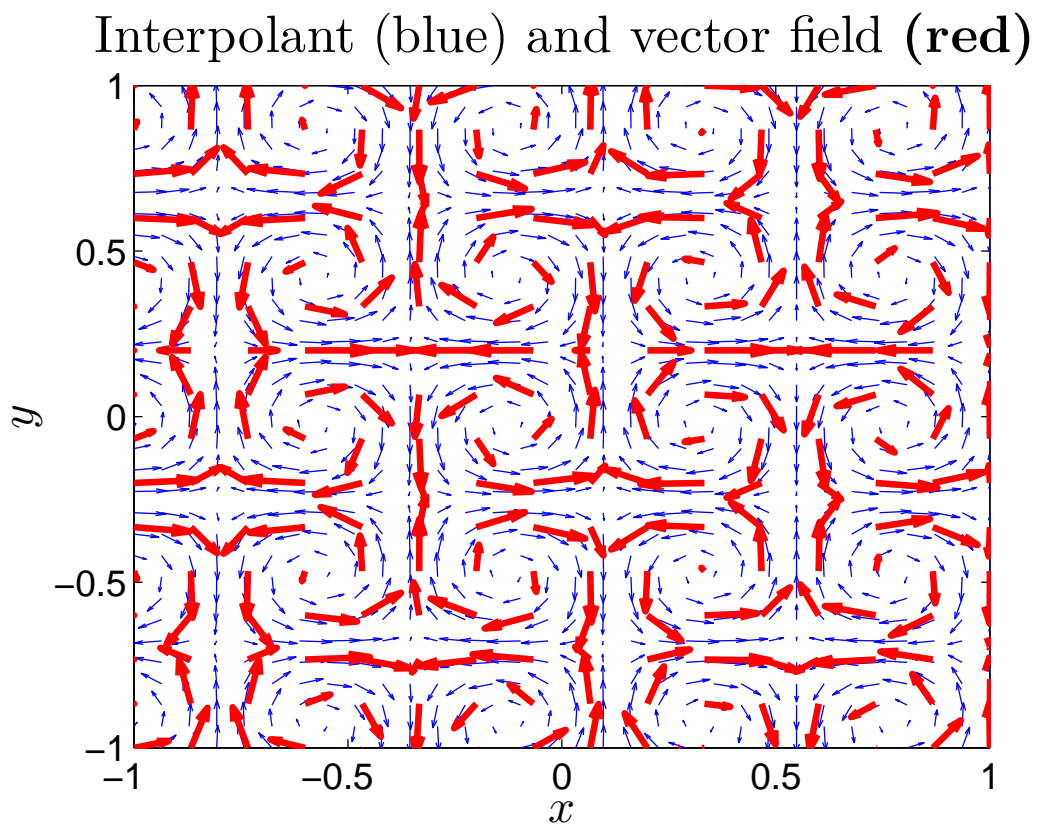

Figure 2: Divergence-free interpolant $($ thin $\longrightarrow$ ) and the vector field at the data sites $($ thick $\longrightarrow$ ) for $N=256$.

Figure 3 shows how the error decays for the $u$-component (horizontal direction) of the vector field using the regular RBF interpolation and the divergence-free RBF method. We see that both methods achieve a precision of $10^{-6}$ when $\sqrt{N}=30$. For larger values of $N$, the error ceases to decrease. This behavior happens because interpolation matrices for both methods, although invertible, become ill-conditioned. Table 1 shows how fast the condition numbers grows.

\begin{tabular}{cll}
\hline \hline & \multicolumn{2}{c}{ cond(A) } \\
\cline { 2 - 3 }$\sqrt{N}$ & divergence-free & regular \\
\hline \hline 3 & $1.52 \times 10^{0}$ & $1.11 \times 10^{0}$ \\
5 & $1.12 \times 10^{2}$ & $1.87 \times 10^{1}$ \\
7 & $2.48 \times 10^{5}$ & $2.82 \times 10^{3}$ \\
9 & $4.36 \times 10^{9}$ & $2.03 \times 10^{6}$ \\
11 & $2.62 \times 10^{14}$ & $5.06 \times 10^{9}$ \\
13 & $7.21 \times 10^{18}$ & $3.36 \times 10^{13}$ \\
15 & $3.05 \times 10^{18}$ & $8.69 \times 10^{16}$ \\
\hline \hline
\end{tabular}

Table 1: Condition number of the interpolation matrices of the divergence-free and regular RBF method.

Note that the interpolation matrix is $N \times N$ for the traditional method and $2 N \times 2 N$ for the divergencefree method, which makes the generation of divergence-free interpolants more computationally expensive. Moreover, we see in Table 1 that the interpolation matrix for the divergence-free method has a larger condition number than for the regular case when the same shape parameter is used.

In Table 2, we present the error decay for the partial derivative of the $u$-component of the vector field. Note that we get a slightly more accurate result using the divergence-free interpolant when compared with the traditional RBF interpolant. The same feature is observed for local divergence-free interpolants in Section 3.2 .

Notice that we only comment on the $u$-component of the field. Due to symmetry, the same observations hold for the vertical component of the vector field $(v)$, with $v_{y}$ behaving analogously to $u_{x}$ and $v_{x}$ to $u_{y}$. 


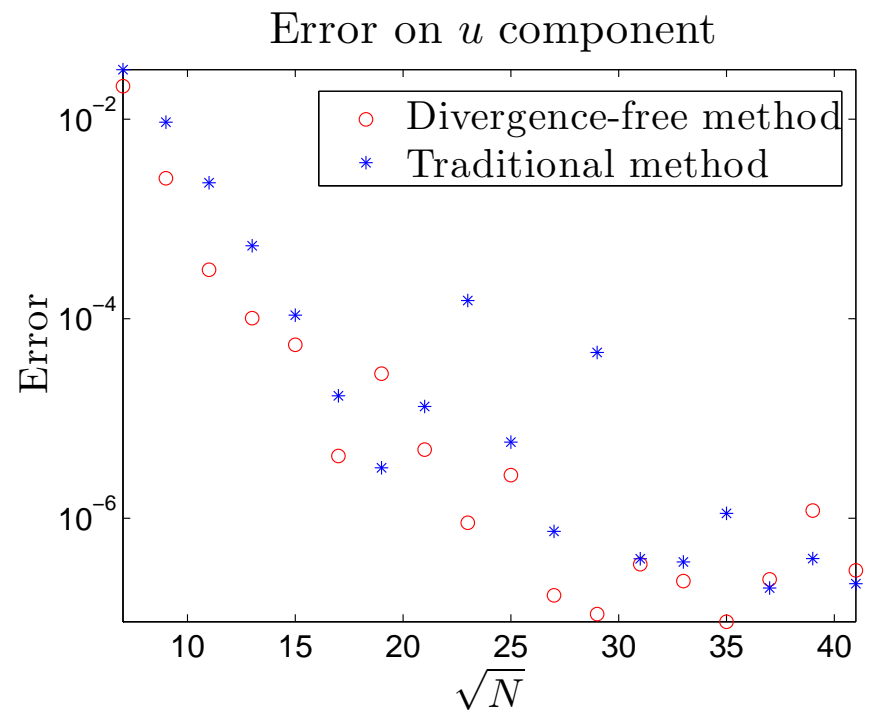

Figure 3: Error decay for the first component of the vector field for both methods of interpolation.

\begin{tabular}{clllll}
\hline \hline & \multicolumn{2}{c}{$u_{x}$} & & \multicolumn{2}{c}{$u_{y}$} \\
\cline { 2 - 3 } \cline { 5 - 6 }$\sqrt{N}$ & divergence-free & regular & & divergence-free & regular \\
\hline \hline 3 & $1.23 \times 10^{-1}$ & $1.26 \times 10^{-1}$ & & $7.08 \times 10^{-1}$ & $6.17 \times 10^{-1}$ \\
5 & $1.91 \times 10^{-1}$ & $1.63 \times 10^{-1}$ & & $8.20 \times 10^{-1}$ & $7.97 \times 10^{-1}$ \\
7 & $4.52 \times 10^{-3}$ & $7.09 \times 10^{-3}$ & & $1.26 \times 10^{-1}$ & $3.46 \times 10^{-2}$ \\
9 & $7.91 \times 10^{-5}$ & $1.89 \times 10^{-3}$ & & $4.94 \times 10^{-3}$ & $9.23 \times 10^{-3}$ \\
11 & $1.46 \times 10^{-6}$ & $4.06 \times 10^{-4}$ & & $4.08 \times 10^{-4}$ & $1.98 \times 10^{-3}$ \\
13 & $3.06 \times 10^{-8}$ & $5.13 \times 10^{-5}$ & & $9.27 \times 10^{-5}$ & $2.50 \times 10^{-4}$ \\
15 & $1.03 \times 10^{-7}$ & $4.16 \times 10^{-6}$ & & $7.31 \times 10^{-6}$ & $2.03 \times 10^{-5}$ \\
\hline \hline
\end{tabular}

Table 2: Error decay for the derivatives of the first component of the vector field $(u)$. Note that the divergence-free interpolant provides a slightly more accurate results.

\subsection{Local divergence-free numerical results}

We now focus on the accuracy of local approximations. Our first example uses a rectangular $3 \times 3$ grid. The derivatives are evaluated at the center point and compared to the exact derivatives of $\boldsymbol{f}$ (defined in (9)).

Figure 4 shows two experiments, one using degree $n=2$ and the other one using degree $n=3$ for the polynomial expansion of the stream function (8). In the case $n=2$, there are 8 unknown coefficients in (8) and 18 data values to fit in the $3 \times 3$ grid ( 9 for $u$ and 9 for $v$ ), resulting in a $18 \times 8$ least squares system that is full rank. For $n=3$ the size of the linear system is $18 \times 15$ and is rank deficient, with 14 linearly independent columns.

These experiments illustrate how the degree can change the rate of convergence of the method. For $n=3, u_{x}$ is accurate to fourth order and $u_{y}$ to second. For $n=2$, on the other hand, both directions are second order accurate. Note that we have similar results for the partial derivatives of the second component of $\boldsymbol{f}$. One possible explanation for this improvement in the convergence rates is that $u_{x}$ and $v_{y}$ are the derivative components that appear in the divergence operator. Writing

$$
u(x, y)=u_{1}(x, y)+u_{2}(y) \text { and } v(x, y)=v_{1}(x, y)+v_{2}(x)
$$

we have that the divergence-free condition is satisfied whenever $\left(u_{1}\right)_{x}+\left(v_{1}\right)_{y}=0$, independently of the functions $u_{2}$ and $v_{2}$. Because our stencil is rectangular and we are using three points in each direction, the derivatives of $u_{2}$ and $v_{2}$ can only be accurate to second order, while $u_{1}$ and $v_{1}$ are accurate to fourth 

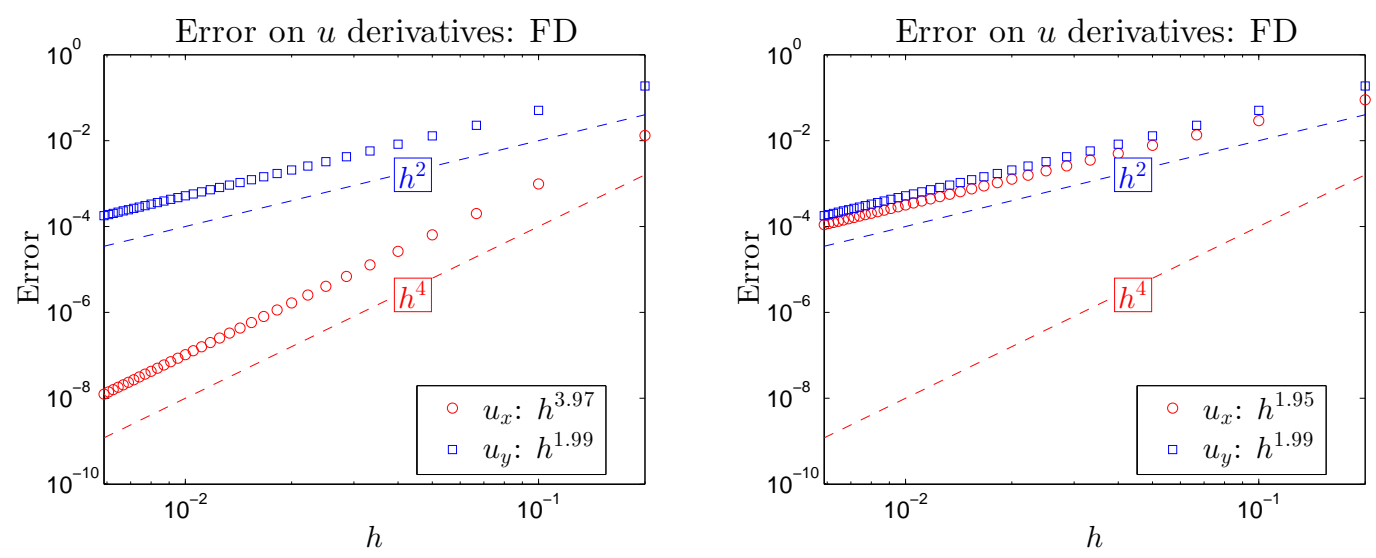

Figure 4: $u_{x}$ and $u_{y}$ denote the approximation of the partial derivatives of the first component of $\boldsymbol{f}$ using nine point stencils. The right picture uses a polynomial of degree $n=2$ and the left picture degree $n=3$.

order in the $x$ and $y$ directions respectively.

Figure 5 shows the error decay for the same numerical experiment, but using the RBF-FD method. The divergence-free RBF-FD method has the same order decay of the divergence-free FD method. One difference is that RBF-FD does not require the solution of a least squares problem to find a divergence-free approximation, that is, an interpolant is guaranteed to exist.

It is important to point out that for the polynomial finite differences method we need to choose an adequate degree in order to obtain faster convergence rates for the derivatives. For example, for a $3 \times 3$ stencil, $n=3$ is the smallest degree for fourth order convergence in $u_{x}$ and $v_{y}$ (and larger degrees will not improve accuracy, see Figure 6). In the scattered node case, the choice of degree is less obvious. Figure 6 displays the error decay for a fixed number of points as the degree $n$ of the stream function is increased. For the scattered nodes case, the error does not decrease for degrees larger then 5 when using a 9 point approximation. Notice that the linear system to be solved in this case is $18 \times 35$ and the coefficients of (8) are chosen as the basic solution computed by Matlab's backslash command.

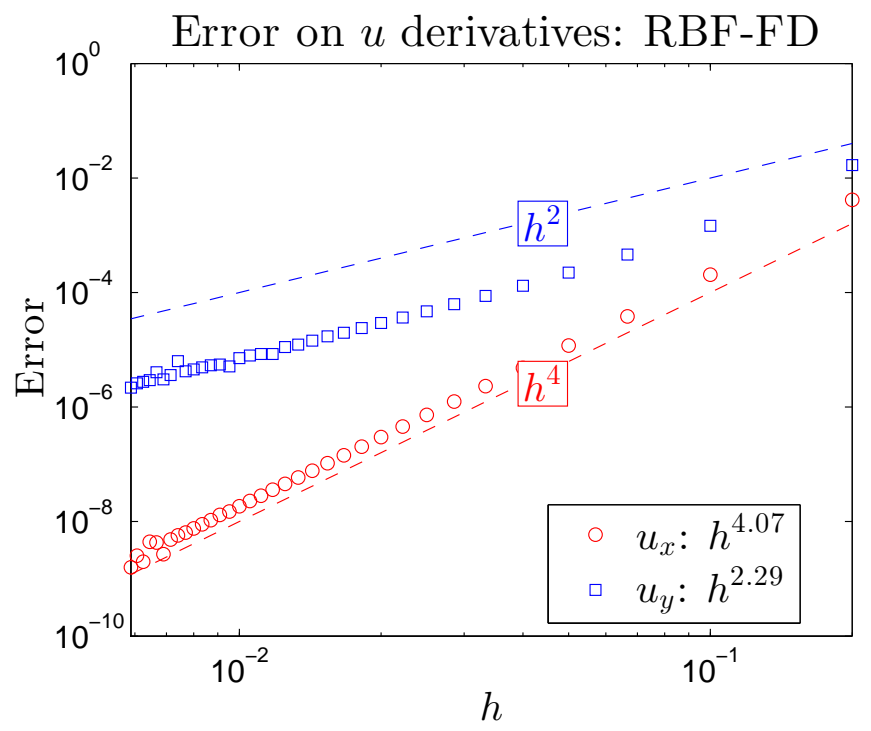

Figure 5: Error decay for the derivatives of $u$ using divergence-free RBF-FD method on nine point stencils. The dashed lines represent the decay of a $2^{\text {nd }}$ and a $4^{\text {th }}$ order methods.

We point out that accuracy results are significantly different for scattered nodes. Our next experiment shows that neither direction is necessarily favored (in contrast to rectangular grids). We generate $N$ 

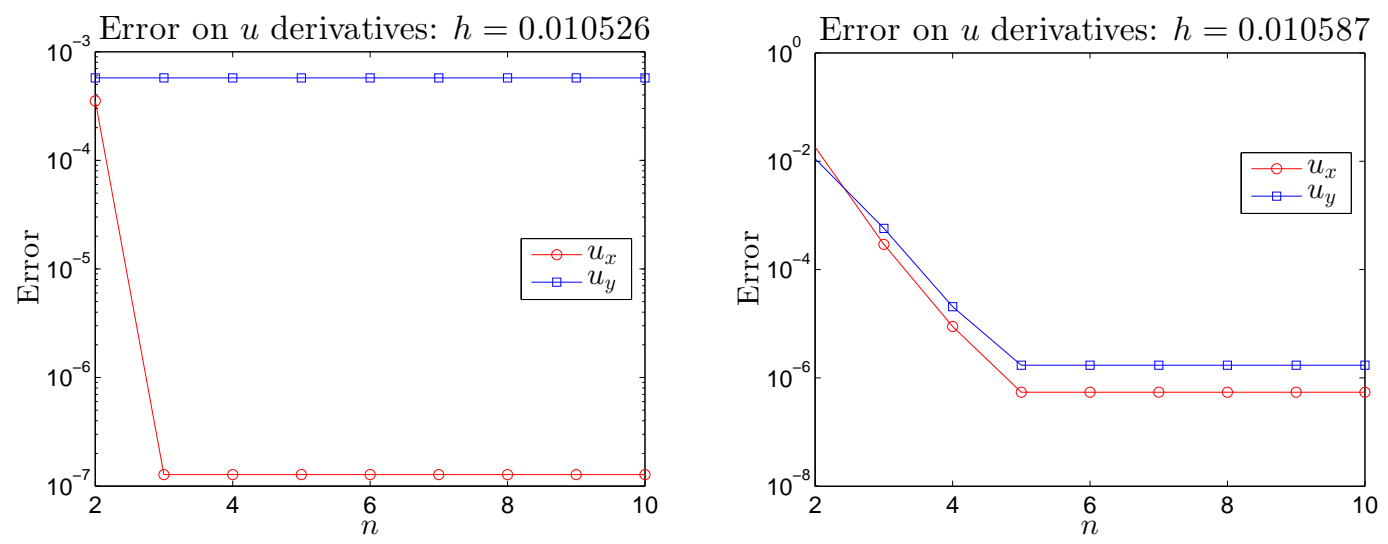

Figure 6: Error decay as a function of the polynomial degree $n$ for fixed number of points. Left: Using a $3 \times 3$ rectangular stencil. Right: Using 9 scattered points. For the rectangular grid case, polynomial degrees larger than 3 do not improve the approximation, while for the unstructured case, degrees larger than 5 saturate the approximation.
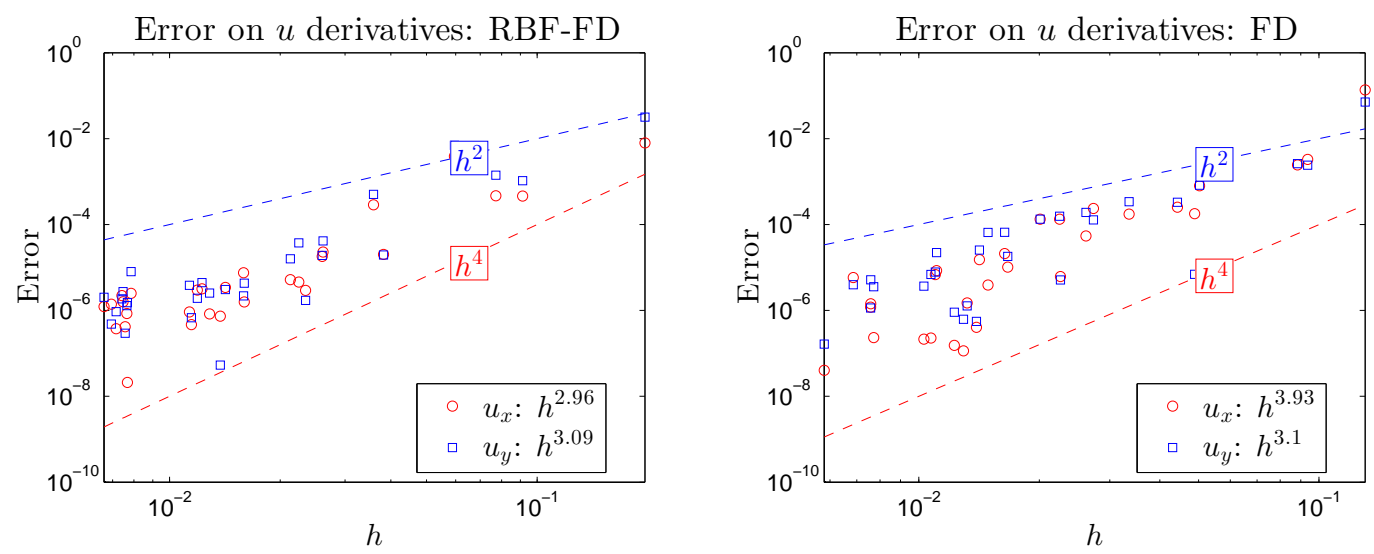

Figure 7: Error decay for divergence-free RBF-FD method on unstructured grids. Left: Divergence-free RBF-FD; Right: Divergence-free polynomial FD using $n=5$ for the degree of the stream function (8).

random points uniformly distributed in $[-1,1]^{2}$ and then select the closest 8 points to where we want to approximate the derivatives. Those 9 points are used to create our local divergence-free interpolant for the RBF and polynomial cases.

Figure 7 shows the error decay for the derivative of the first component of the vector field for both methods. We note that both methods seem to have a convergence rate between second and fourth order. The plot in Figure 7 shows the error as a function of the fill distance

$$
h=\sup _{\boldsymbol{x} \in \Omega} \min _{\boldsymbol{x}_{j} \in \mathcal{X}}\left\|\boldsymbol{x}-\boldsymbol{x}_{j}\right\|_{2}
$$

where $\Omega=[-1,1]^{2}$ and $\mathcal{X}$ is the set of interpolation points. Notice that the effective convergence rate is somewhere between fourth and second orders.

\subsection{Lebesgue constants and the Kosloff 8 Tal-Ezer map}

It is well known that polynomial interpolation on equally spaced nodes suffers from the Runge phenomenon - wild oscillations near the boundaries of the domain when certain analytic functions are interpolated. Classical RBF interpolation with smooth global kernels are also susceptible to this phenomenon [12, 28, 29]. This is illustrate in Figure 8, where the RBF interpolant of $f(x)=1 /\left(1+25 x^{2}\right)$ is shown for decreasing values of shape parameters. As mention before, the RBF interpolant approaches the Lagrange interpolant on a given set of nodes as $\varepsilon \rightarrow 0$, therefore the Runge phenomenon is expected on equispaced points. Associated to this phenomenon is the sensitivity of the interpolation process. That 
is, even for functions where convergence should take place in theory, the exponential ill-conditioning of the interpolation operator causes divergence in floating point arithmetic.
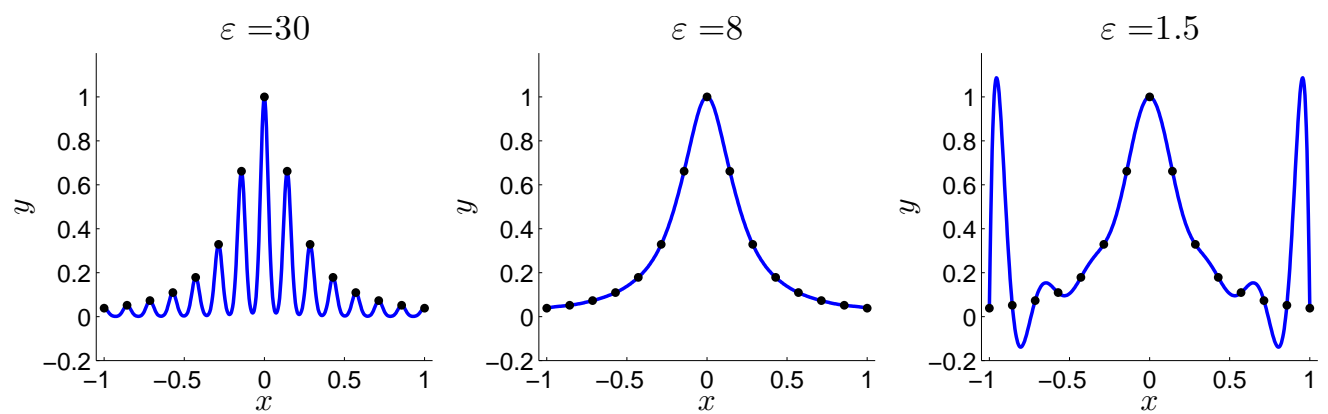

Figure 8: Gaussian RBF interpolant of $f(x)=1 /\left(1+25 x^{2}\right)$ on 21 equally spaced points. The Runge phenomenon is clearly noticeable for $\varepsilon=1.5$.

In this section we explore the sensitivity of the interpolation operator for the divergence-free method to perturbations on the data. To do so, we analyze the values of the Lebesgue constants. To be precise, we defined the Lebesgue constants as

$$
\Lambda_{N}=\sup _{\substack{\boldsymbol{f} \in L^{\infty}(\Omega) \\\|\boldsymbol{f}\|_{N, \infty} \neq 0}}\left\{\frac{\|\boldsymbol{t}\|_{\infty}}{\|\boldsymbol{f}\|_{N, \infty}}\right\},
$$

where $\boldsymbol{t}$ is the divergence-free interpolant at $N$ grid points, $\Omega$ is the approximation domain, $\|\boldsymbol{t}\|_{\infty}$ is the sup norm over $\Omega$, and $\|\boldsymbol{f}\|_{N, \infty}$ is the sup norm of the values of $\boldsymbol{f}$ on the grid. Because $\boldsymbol{f}$ is a vector field, we defined these two norms as the maximum of the norms over each component.

We compute the Lebesgue constant numerically using an equivalent expression (for the 2D case),

$$
\Lambda_{N}=\max _{\boldsymbol{x} \in \Omega}\left(\sum_{i=1}^{N}\left|\boldsymbol{\varphi}_{i}^{u}(\boldsymbol{x})\right|+\sum_{i=1}^{N}\left|\boldsymbol{\varphi}_{i}^{v}(\boldsymbol{x})\right|\right),
$$

where the functions $\boldsymbol{\varphi}_{i}^{u, v}$ are the cardinal functions on the nodes $\boldsymbol{x}_{j}$, i.e. $\boldsymbol{\varphi}_{i}^{u, v}\left(\boldsymbol{x}_{j}\right)=[0,0]^{T}$ if $i \neq j$, $\boldsymbol{\varphi}_{i}^{u}\left(\boldsymbol{x}_{i}\right)=[1,0]^{T}$, and $\boldsymbol{\varphi}_{i}^{v}\left(\boldsymbol{x}_{i}\right)=[0,1]^{T}$. Figure 9 shows two cardinal functions for divergence-free RBF interpolation using shape parameter $\varepsilon=2$ in a $3 \times 3$ stencil.

Figure 10 displays the growth of the Lebesgue constant for different values of shape parameter of the divergence-free RBF interpolant described in Section 2.2 and the polynomial divergence-free interpolant described in Section 2.3 when using global stencils. Unsurprisingly, the polynomial method has the largest
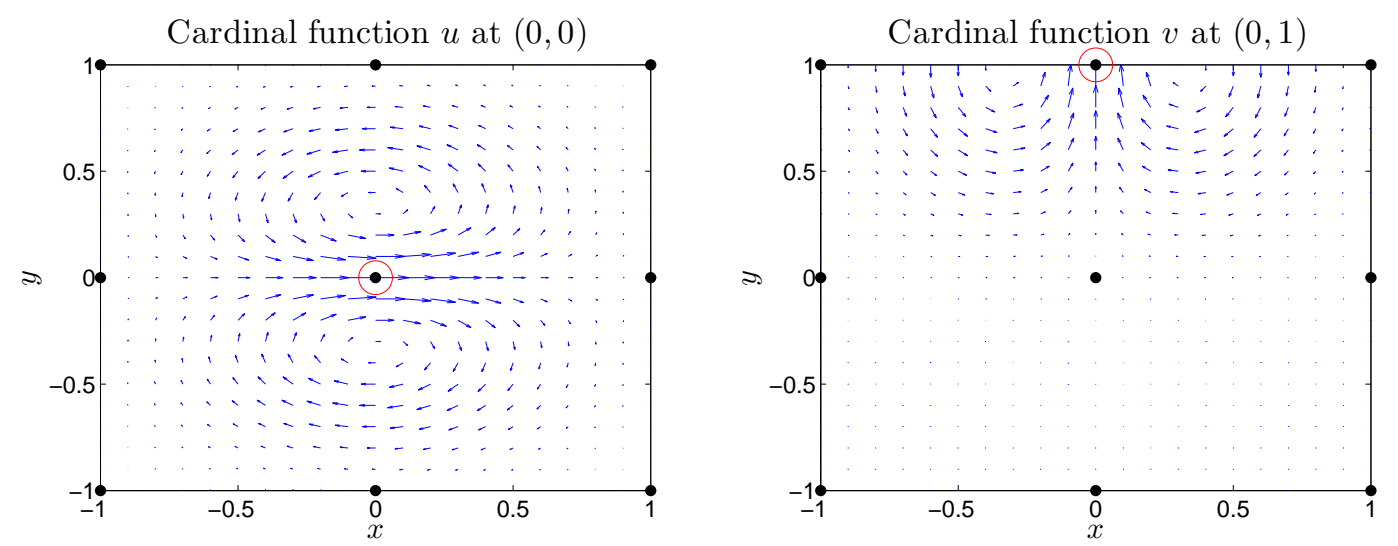

Figure 9: Cardinal functions for divergence-free RBF interpolation with $\varepsilon=2$ in a $3 \times 3$ stencil. Left: $u=1$ at $(0,0)$. Right: $v=1$ at $(0,1)$. 
Lebesgue constant values. Polynomial approximations are notoriously ill-conditioned for interpolation on equispaced nodes ([31]) and similar behavior is observed in the divergence-free case.

Our experiments in Section 4 indicate that the flat limit $(\varepsilon \rightarrow 0)$ of RBF divergence-free interpolants is also a polynomial. Consequently, we see that for small $\varepsilon$, divergence-free RBF approximations also have large Lebesgue constants, while larger values of $\varepsilon$ lead to better conditioned approximations.

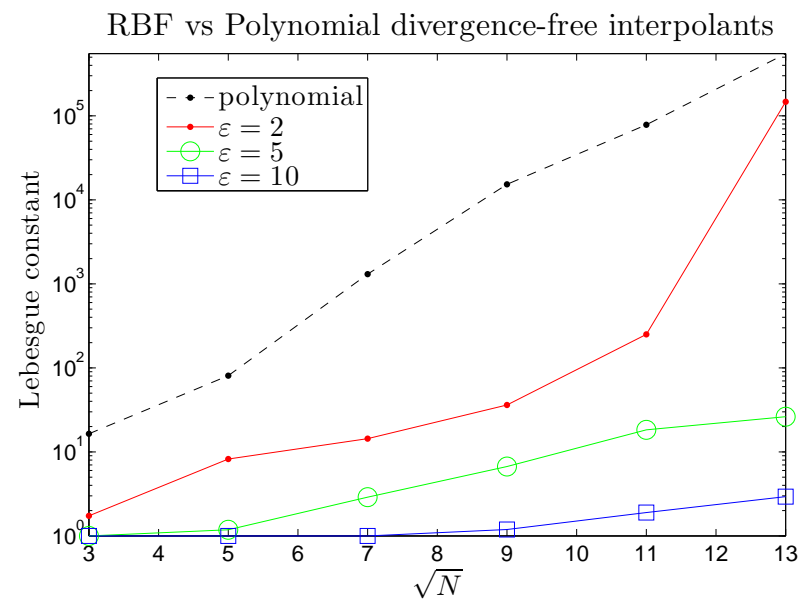

Figure 10: Lebesgue constant growth for divergence-free methods.

One can improve the values of the Lebesgue constant using a mapping technique. In [28], the Kosloff \& Tal-Ezer mapping

$$
x^{k t e(\alpha)}:=\frac{\arcsin \left(\alpha x_{j}^{c h e b}\right)}{\arcsin (\alpha)}, \quad j=1, \ldots, N,
$$

where $x_{j}^{c h e b}=\cos (\pi(j-1) /(N-1))$. Here we use the same parameter $\alpha$ in each spatial direction. The $\mathrm{K}-\mathrm{T}$-E mapping, introduced in [20], maps Chebyshev points into more evenly spaced ones as $\alpha \rightarrow 1$. As $\alpha \rightarrow 0$ the mapping becomes the identity. By choosing $\alpha \in(0,1)$ one can tune the clustering of nodes near the boundaries of the domain.

In Table 3 we show the values of the optimized Lebesgue constants together with the values of the corresponding mapping parameters $\alpha$. For the RBF case, it can be observed that mapping nodes greatly improves the Lebesgue constant. For the polynomial case, using Chebyshev points for both spacial directions leads to small Lebesgue constants (around 40 in our experiments), indicating that this node distribution is also a good choice for divergence-free polynomial bases.

\begin{tabular}{|c|c|c|c|c|c|c|c|c|c|}
\hline \multirow[b]{2}{*}{$\sqrt{N}$} & \multicolumn{3}{|c|}{$\varepsilon=2$} & \multicolumn{3}{|c|}{$\varepsilon=5$} & \multicolumn{3}{|c|}{$\varepsilon=10$} \\
\hline & $\Lambda(\alpha=1)$ & $\min _{\alpha} \Lambda$ & $\alpha_{\min }$ & $\Lambda(\alpha=1)$ & $\min _{\alpha} \Lambda$ & $\alpha_{\min }$ & $\Lambda(\alpha=1)$ & $\min _{\alpha} \Lambda$ & $\alpha_{\min }$ \\
\hline 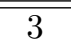 & (1.7 & 1.7 & 0.788 & "1.0 & (1.0 & 1.000 & "1.0 & (1.0 & 1.000 \\
\hline 5 & 8.2 & 5.8 & 0.817 & 1.2 & 1.6 & 0.624 & 1.0 & 1.0 & 0.797 \\
\hline 7 & 14.4 & 13.6 & 0.980 & 2.9 & 2.9 & 0.973 & 1.0 & 1.6 & 0.690 \\
\hline 9 & 36.2 & 24.0 & 0.992 & 6.7 & 5.4 & 0.964 & 1.2 & 1.6 & 0.974 \\
\hline 11 & 250.4 & 44.4 & 0.967 & 18.3 & 15.7 & 0.949 & 1.9 & 1.9 & 0.998 \\
\hline 13 & $1.2 \times 10^{4}$ & 166.9 & 0.950 & 26.3 & 24.7 & 0.998 & 2.9 & 2.9 & 1.000 \\
\hline
\end{tabular}

Table 3: Value of the Lebesgue constant $\Lambda$ for equally spaced points $(\alpha=1)$ and optimized nodes (min $\alpha$ ). Lebesgue constants calculated for divergence-free RBF interpolants using $\varepsilon=2,5,10$.

\section{Limit of the divergence-free interpolant as $\varepsilon \rightarrow 0$}

It has been shown in [4, for the unidimensional case, and in [21, 32, 22] for the multivariate case, that the limit of increasingly flat RBF interpolants converge to polynomials. In the multivariate case, the limit 

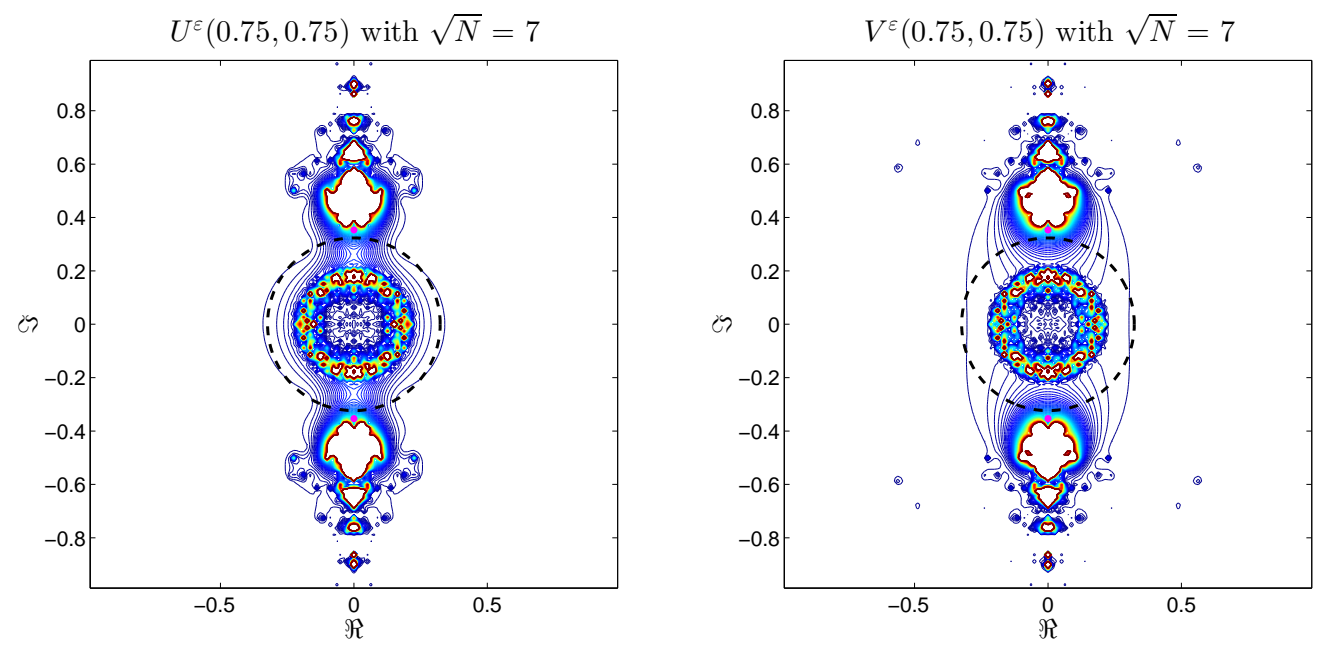

Figure 11: Absolute value of $u$ (left) and $v$ (right) at $(0.75,0.75)$ as the shape parameter varies in the complex plane. The main idea to calculate the limit via the contour-Padé algorithm is to evaluate the interpolant for complex values of $\varepsilon$. The limit is calculated via a contour integral, where the contour is chosen (dashed line) to avoid the ill-conditioned region of the interpolation matrix and the poles of the interpolant.

is only guaranteed to exist if the node set is polynomial unisolvent, except for Gaussians (see also [11] for details). In this section we present numerical results that indicate that the flat limit of divergence-free RBF interpolants is also a polynomial. We only show results for two basic functions $\phi$ that define the divergence-free kernel: Gaussians (G) and generalized inverse multiquadrics with $\beta=2$ (IM), but similar behavior is expected for other basic functions.

For a small number of nodes, the flat limit can be found using symbolic computations. For more than a few nodes, exact computation grows too complex to provide useful information about the limit. Computations in this case were carried out in double precision. As the shape parameter decreases to zero, the condition number of the interpolation matrix becomes too large for practical computations (even with only 9 points). Fortunately, a technique presented in [10] allows the computation of the interpolant at the limit $\varepsilon \rightarrow 0$. The main idea is to consider $\varepsilon$ (the shape parameter) a complex variable. The RBF interpolant is an analytic function of $\varepsilon$ near $\varepsilon=0$. We can then compute the interpolant at the flat limit by evaluating a contour integral around the origin. The computational cost of this technique is too high for large scale problems, but works well for the problem at hand. This idea is illustrated in Figure 11.

Using the contour integral method, we evaluate the flat limit interpolant at several points. To verify whether the limit is a polynomial or not, we interpolate the values using a tensor product of Chebyshev polynomials. In all our test cases only a small number of nonzero coefficients are needed for this representation. The non-zero coefficients are $\mathcal{O}(1)$ while the zero coefficients are about machine precision. We use the two-dimensional capability of chebfun [30, 5, 34] to obtain the polynomial representation.

Figure 12 shows the divergence-free interpolant for a five point stencil with data given by $\boldsymbol{f}(0,0)=$ $[1,0]^{T}$ and $\boldsymbol{f}(1,0)=\boldsymbol{f}(0,1)=\boldsymbol{f}(-1,0)=\boldsymbol{f}(0,-1)=[0,0]^{T}$. In this case we were able to find the interpolant analytically, which allowed us to compare with the chebfund approximation and see that they agree up to machine precision. The expression for the limit is:

$$
\boldsymbol{p}_{G}(x, y)=\left[\begin{array}{c}
-x^{2}-y^{2}+1 \\
2 x y
\end{array}\right]=\boldsymbol{p}_{I M}(x) .
$$

Note that the limit is the same for both basic functions (G and IM), however this is not always the case.

For the interpolant using $\boldsymbol{f}(0,0)=[1,0]^{T}$ and $[0,0]^{T}$ for all the other points on a $3 \times 3$ rectangular 

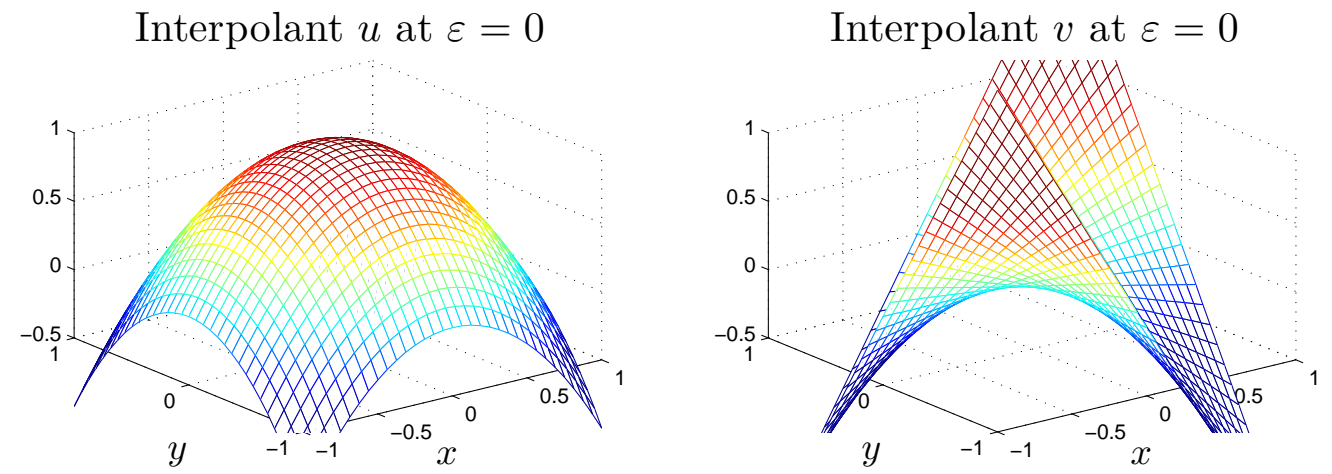

Figure 12: Divergence-free RBF interpolant for $\boldsymbol{f}(0,0)=[1,0]^{T}$ and $\boldsymbol{f}(0,1)=\boldsymbol{f}(1,0)=\boldsymbol{f}(0,-1)=\boldsymbol{f}(-1,0)=[0,0]^{T}$, using the contour-Padé algorithm. In this case, the difference between analytically and numerically computed limits is $\mathcal{O}\left(10^{-15}\right)$.

stencil we obtain

$$
\begin{aligned}
\boldsymbol{p}_{G}^{*}(x, y) & =\left[\begin{array}{c}
\frac{2}{3} x^{4}+x^{2} y^{2}-\frac{5}{3} x^{2}-y^{2}+1 \\
-\frac{8}{3} x^{3} y-\frac{2}{3} x y^{3}+\frac{10}{3} x y
\end{array}\right] \\
\boldsymbol{p}_{I M}^{*}(x, y) & =\left[\begin{array}{c}
\frac{2}{3} x^{4}+x^{2} y^{2}-\frac{5}{3} x^{2}+\frac{47}{429} y^{4}-\frac{476}{429} y^{2}+1 \\
-\frac{8}{3} x^{3} y-\frac{2}{3} x y^{3}+\frac{10}{3} x y
\end{array}\right] .
\end{aligned}
$$

Here the interpolation points are $\{(0,0),(1,0),(0,1),(1,1),(-1,0),(0,-1),(-1,-1),(1,-1),(-1,1)\}$. These interpolants are displayed in Figure 13 . For this case we were not able to acquire the interpolant analytically, however the approximation via chebfun2 of the numerical limit given by the contour-Padé algorithm is a polynomial (we truncate the terms of the polynomial expansion that have coefficients numerically zero). Moreover, here we see that the limits can be different for different basic functions. In this case, the difference between $\boldsymbol{p}_{G}$ and $\boldsymbol{p}_{I M}$ is of the order $10^{-2}$ in the square $[-1,1]^{2}$ but grows larger outside this region.

\section{Three-dimensional vector fields}

We now briefly discuss the three-dimensional case. The RBF kernel (5) is two-dimensional, and to deal with three-dimensional fields, we use the divergence-free kernel introduced by Narcowich and Ward in [26]. The divergence-free interpolant is similar to (4):

$$
\boldsymbol{t}(\boldsymbol{x})=\sum_{k=1}^{N} \Psi\left(\boldsymbol{x}, \boldsymbol{x}_{k}\right) \boldsymbol{s}_{k}
$$

with $\boldsymbol{s}_{k}=\left(s_{k}^{1}, s_{k}^{2}, s_{k}^{3}\right)$ the coefficient vector, but where

$$
\begin{aligned}
\Psi(\boldsymbol{x}, \boldsymbol{y}) & =\left(-\Delta I+\nabla \nabla^{T}\right) e^{-\varepsilon\|\boldsymbol{x}-\boldsymbol{y}\|^{2}} \\
& =\left[\left(4 \varepsilon-4 \varepsilon^{2}\|\boldsymbol{x}-\boldsymbol{y}\|^{2}\right) I+4 \varepsilon^{2}(\boldsymbol{x}-\boldsymbol{y})(\boldsymbol{x}-\boldsymbol{y})^{T}\right] e^{-\varepsilon\|\boldsymbol{x}-\boldsymbol{y}\|^{2}}, \quad \varepsilon>0,
\end{aligned}
$$

with $\nabla$ is the gradient operator, $\Delta$ is the Laplacian operator and $I$ the $3 \times 3$ identity matrix. It is not hard to show that the columns of the matrix $\Psi(\boldsymbol{x}, \boldsymbol{y})$ have zero divergence with respect to $\boldsymbol{x}$. Using the given data, $d_{j}^{\bar{l}}=t_{\bar{l}}\left(\boldsymbol{x}_{j}\right)$, we have

$$
d_{j}^{\bar{l}}=\sum_{k=1}^{N} \sum_{l=1}^{3} s_{k}^{l} \Psi_{\overline{l l}}\left(\boldsymbol{x}_{j}, \boldsymbol{x}_{k}\right)=\sum_{l=1}^{3}\left(A^{\bar{l} \times l} \boldsymbol{s}^{l}\right)_{j},
$$




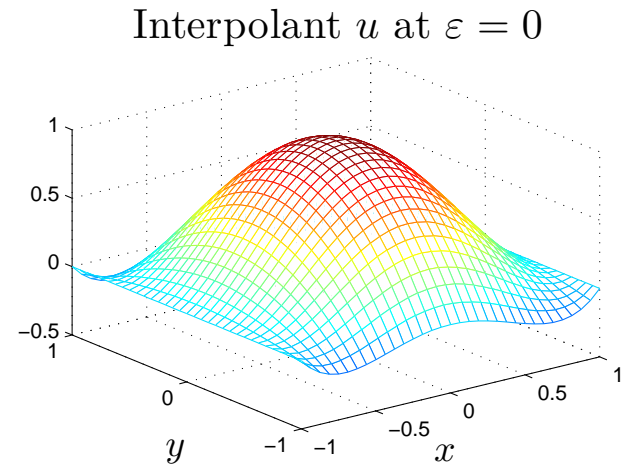

Interpolant $u$ at $\varepsilon=0$

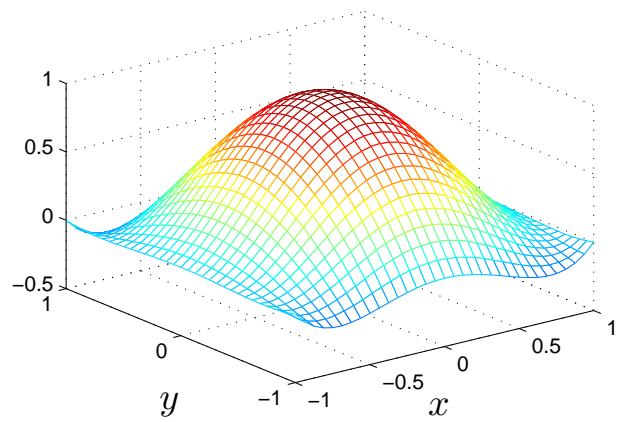

Interpolant $v$ at $\varepsilon=0$

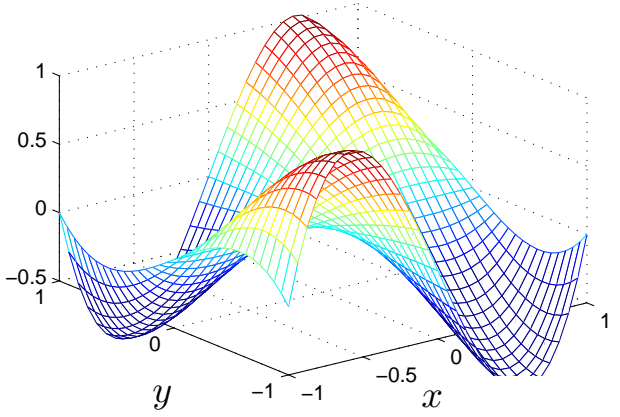

Interpolant $v$ at $\varepsilon=0$

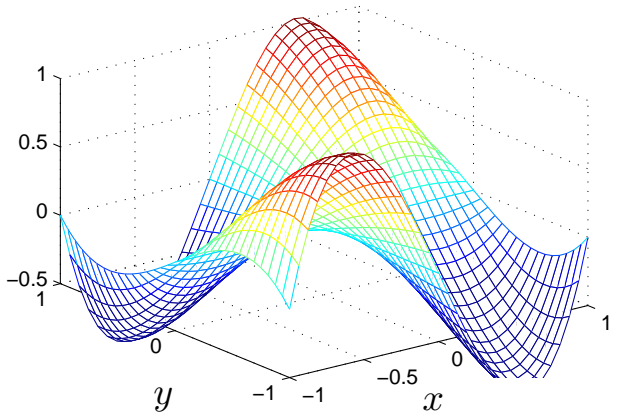

Figure 13: Divergence-free RBF interpolant for $\boldsymbol{f}(0,0)=[1,0]^{T}$ and $\boldsymbol{f}(0,1)=\boldsymbol{f}(1,0)=\boldsymbol{f}(0,-1)=\boldsymbol{f}(-1,0)=\boldsymbol{f}(1,1)=$ $\boldsymbol{f}(-1,1)=\boldsymbol{f}(1,-1)=\boldsymbol{f}(-1,-1)=[0,0]^{T}$. Top: Gaussians. Bottom: inverse multiquadrics. The difference between the top and bottom interpolants is approximately $2.7 \times 10^{-2}$ on $[-1,1]^{2}$.

where $A_{j k}^{\bar{l} \times l}=\Psi_{\overline{l l}}\left(\boldsymbol{x}_{j}, \boldsymbol{x}_{k}\right), \boldsymbol{d}^{\bar{l}}=\left[d_{1}^{\bar{l}}, \ldots, d_{N}^{\bar{l}}\right]^{T}$ and $\boldsymbol{s}^{l}=\left[s_{1}^{l}, \ldots, s_{N}^{l}\right]^{T}$ for $\bar{l}, l=1,2,3$ and $j, k=1, \ldots, N$. Defining

$$
A=\left[\begin{array}{lll}
A^{1 \times 1} & A^{1 \times 2} & A^{1 \times 3} \\
A^{2 \times 1} & A^{2 \times 2} & A^{2 \times 3} \\
A^{3 \times 1} & A^{3 \times 2} & A^{3 \times 3}
\end{array}\right], \quad \boldsymbol{c}=\left[\begin{array}{c}
\boldsymbol{s}^{1} \\
\boldsymbol{s}^{2} \\
\boldsymbol{s}^{3}
\end{array}\right] \quad \text { and } \quad \boldsymbol{d}=\left[\begin{array}{l}
\boldsymbol{d}^{1} \\
\boldsymbol{d}^{2} \\
\boldsymbol{d}^{3}
\end{array}\right]
$$

we can summarize the interpolation conditions in the linear system $\boldsymbol{d}=A \boldsymbol{c}$, which was proved to have a unique solution in [26]. To acquire derivative matrices, we follow the same procedure used in Section 2.1.

To test the local accuracy of this kernel we use the function $\boldsymbol{f}(x, y, z)=\nabla \times \boldsymbol{\psi}(x, y, z)$, where $\boldsymbol{\psi}=\left(\psi_{1}, \psi_{2}, \psi_{3}\right)$ and

$$
\begin{aligned}
& \psi_{1}(x, y, z)=\sin \left((x-1)^{2}+(y-1)^{2}+(z-1)^{2}\right) \\
& \psi_{2}(x, y, z)=\cos \left((x-0.5)^{2}+(y-0.5)^{2}+(z-0.5)^{2}\right) \\
& \psi_{3}(x, y, z)=\sin \left((x+0.5)^{2}+(y-1)^{2}+(z+1)^{2}\right) .
\end{aligned}
$$

Figure 14 shows the error decay for evenly spaced $3 \times 3 \times 3$ Cartesian stencils. Just as in the twodimensional case, we have an increase in the convergence rate of the derivatives present in the divergence operator $\left(u_{x}, v_{y}\right.$ and $\left.w_{z}\right)$, however this precision is due to the disposition of our grid points. In Figure 15 we repeat the experiment using an unstructured grid formed by the origin and the 26 nearest points. As in the $2 \mathrm{D}$ case, a convergence rate between second and fourth order is observed. For both experiments we use shape parameter $\varepsilon=8$.

\section{Concluding remarks}

Divergence-free vector fields can be more accurately represented by solenoidal bases than with independent component-wise approximations. On a rectangular grid, the main gain in accuracy is in the directions of the derivatives present in the divergence operator. Two additional orders of accuracy were 

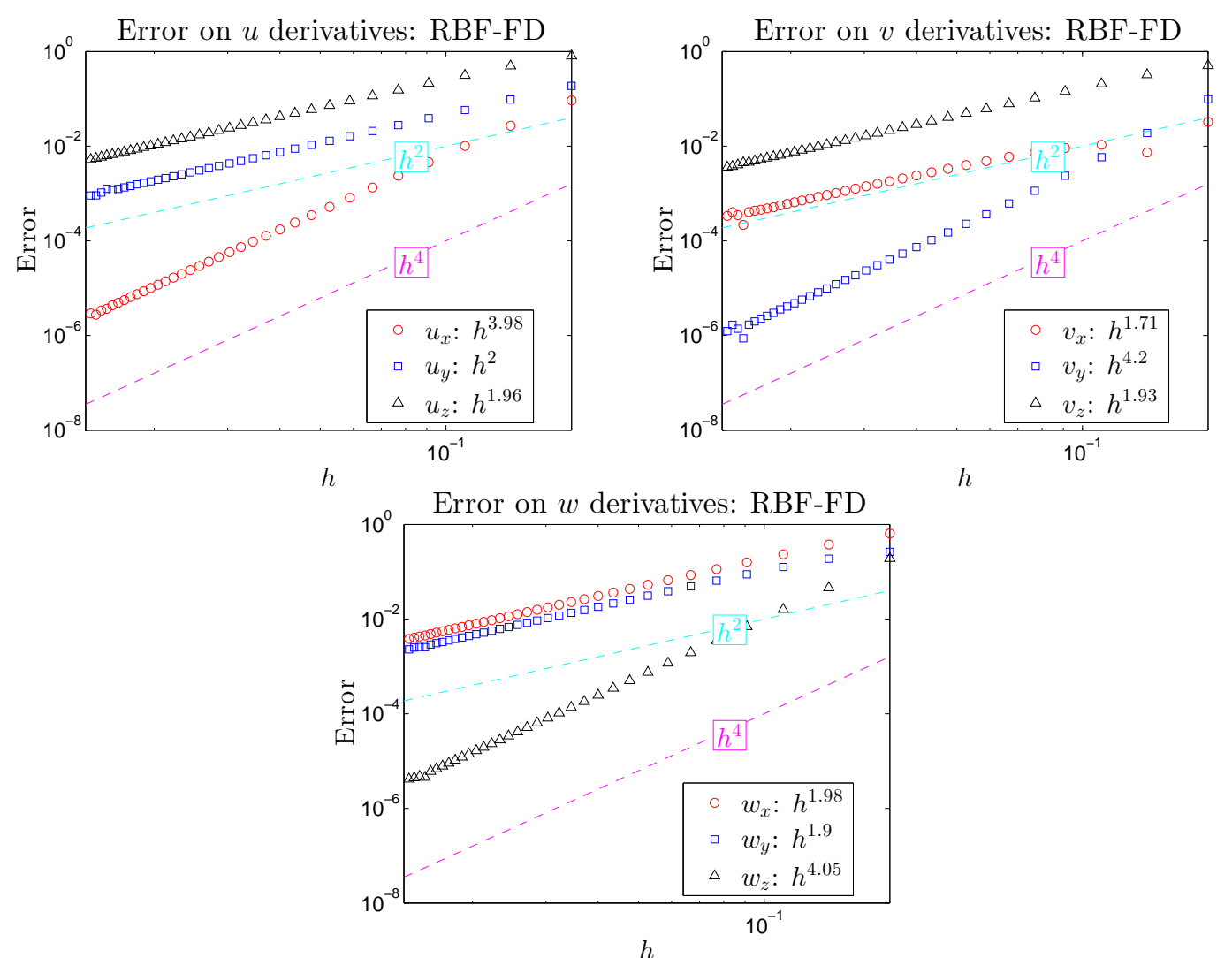

Figure 14: Error in the approximation of the derivatives of a divergence-free vector field using the 3D divergence-free RBF method with Gaussians and $\varepsilon=8$. 


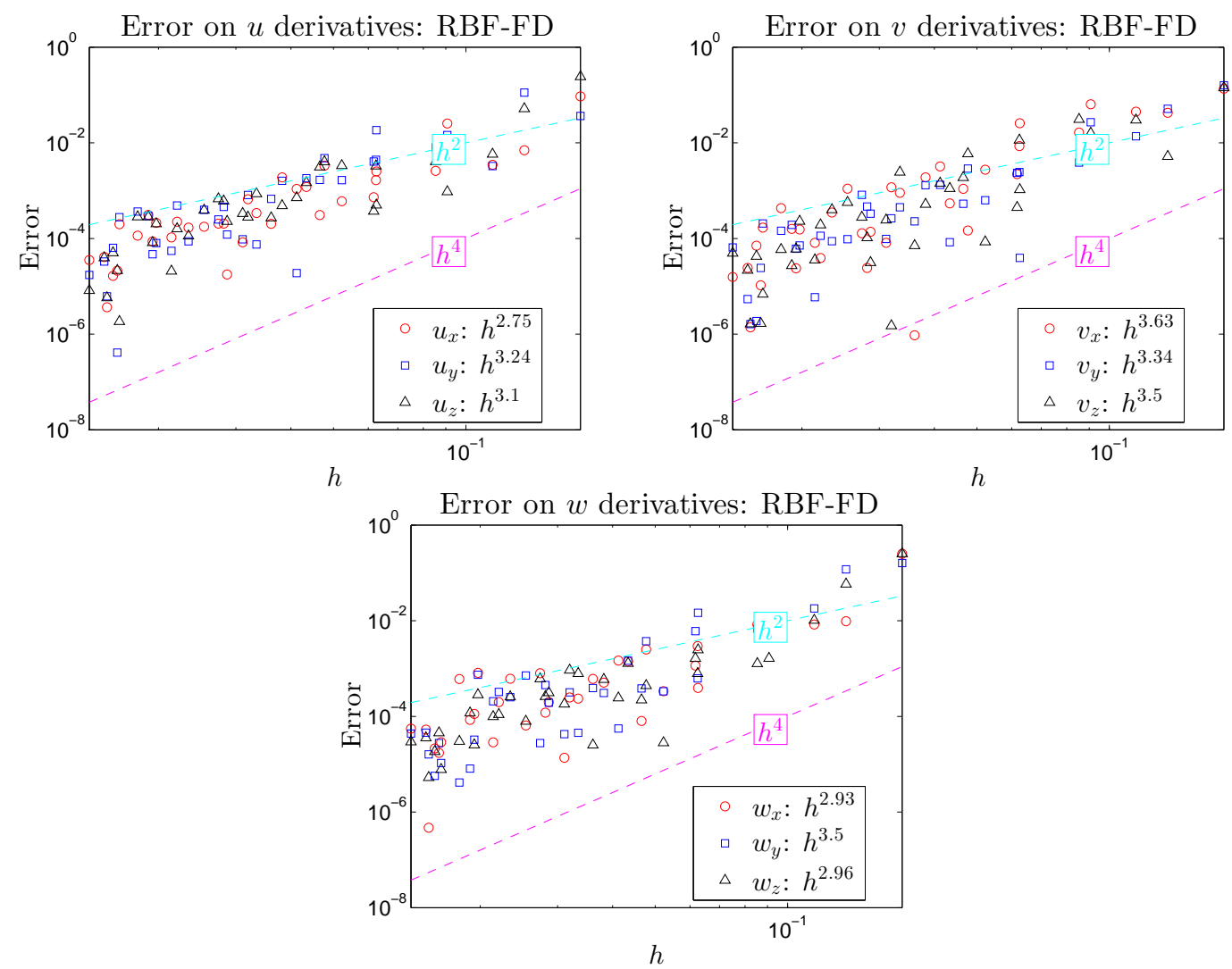

Figure 15: Error on the derivatives of the 3D divergence-free RBF interpolant using unstructured grids and $\varepsilon=8$ (Gaussians). 
observed using RBF kernels in two and three dimensions, as well as in polynomial based approximations in 2D Cartesian stencils. When random or scattered nodes are used, the gain in accuracy is more evenly distributed in all directions. In this case, using a nine-point approximation in $2 \mathrm{D}$ resulted in an effective convergence rate between $\mathcal{O}\left(h^{2}\right)$ and $\mathcal{O}\left(h^{4}\right)$.

We point out that the computational cost for computing a divergence-free interpolant is $\mathcal{O}\left((d N)^{3}\right)$, in the global case and using a direct solver, where $d$ is the vector dimension and $N$ the number of nodes. Using traditional approximations on each vector component, on the other hand, requires $\mathcal{O}\left(N^{3}\right)$, since factorizations need only to be done once and can be reused for the approximation of each entry of the vector. Therefore, for 2D-dimensional problems the cost would be increase by a factor of 8 and for 3 D-dimensional problems by a factor of 27 which is rather expensive and seems to offset gains in accuracy.

The computational cost for solving time dependent PDEs with explicit time-stepping, on the other hand, is dictated by matrix-vector multiplications (if the problem is formulated using differentiation matrices, for instance). At each time step, the cost of calculating all the derivatives of a $2 \mathrm{D}(3 \mathrm{D})$ vector field is $4 \times \mathcal{O}\left(N^{2}\right)\left(9 \times \mathcal{O}\left(N^{2}\right)\right)$ for the traditional method and $2 \times \mathcal{O}\left((2 N)^{2}\right)\left(3 \times \mathcal{O}\left((3 N)^{2}\right)\right)$ for the divergence-free approach. This leads to an increase in computational cost by a factor of 2 (3) due to the larger differentiation matrices of the divergence-free method. Thus, looking back at Table 2, the error between the two approaches seems comparable for a fixed flops budget, however the divergence-free approach guarantees a numerically zero divergence, which is a desirable feature in certain applications.

As for regular RBF approximations, our results indicate that the flat limit $(\varepsilon \rightarrow 0)$ of divergence-free kernels is also a polynomial (in each component). For small problems (less than ten points), the limit can be obtained analytically using symbolic computations depending on the kernel and node distribution. For the more general cases, and larger number of points, the limit can be computed using the contour integral technique introduced in [10]. We have not been able to draw a connection between the limiting polynomial and the polynomial approximation computed using the method presented in Section 2.3 . In some instances, the same limit was obtained with Gaussians and inverse multiquadrics, but like in the standard RBF case [21], the limit can be different polynomials for these two kernels depending on the node distribution.

Our results also show that the condition number of the approximation procedure can be very large on an equispaced Cartesian grid. This is true for both polynomial and smooth divergence-free RBF kernels, although Lebesgue constants do not grow as fast in the latter case if the shape parameter is not close to zero. Clustering nodes more densely near boundaries was shown to lead to significantly smaller Lebesgue constants. The clustering was performed by optimizing the Kosloff-Tal-Ezer mapping (one parameter optimization). Unfortunately, even if Lebesgue constants are of moderate size, condition number of interpolation matrices can still grow exponentially for smooth kernels, a problem that can be addressed by a change of basis using a procedure similar to the RBF-QR method presented in [9, 17.

The source code used to generate the numerical results presented in this article is available at [25.

\section{Acknowledgements}

We thank Grady Wright and Edward Fuselier for several fruitful discussions. We also thank the two anonymous reviewers for their valuable suggestions and comments. This work was supported in part by AFOSR FA9550-12-1-0393.

\section{References}

[1] L. Amodei, M. Benbourhim, A vector spline approximation, J. of Approx. Theory 67 (1991) 51-79.

[2] F. Dodu, C. Rabut, Vectorial interpolation using radial-basis-like functions, Comput. Math. Appl. 43 (2002) 393-411.

[3] F. Dodu, C. Rabut, Irrotational or divergence-free interpolation, Numer. Math. 98 (2004) 477-498.

[4] T.A. Driscoll, B. Fornberg, Interpolation in the limit of increasingly flat radial basis functions, Comput. Math. Appl. 43 (2002) 413-422.

[5] T.A. Driscoll, N. Hale, L.N. Trefethen, Chebfun guide, 2014. 
[6] N. Flyer, E. Lehto, S. Blaise, G.B. Wright, A. St-Cyr, A guide to RBF-generated finite differences for nonlinear transport: Shallow water simulations on a sphere, J. Comput. Phys. 231 (2012) 4078-4095.

[7] B. Fornberg, E. Larsson, N. Flyer, Stable computations with gaussian radial basis functions, SIAM J. Sci. Comput. 33 (2011) 869-892.

[8] B. Fornberg, E. Lehto, Stabilization of RBF-generated finite difference methods for convective PDEs, J. Comput. Phys. 230 (2011) 2270-2285.

[9] B. Fornberg, C. Piret, A stable algorithm for flat radial basis functions on a sphere, SIAM J. Sci. Comput. 30 (2007) 60-80.

[10] B. Fornberg, G. Wright, Stable computation of multiquadric interpolants for all values of the shape parameter, Comput. Math. Appl. 48 (2004) 853-867.

[11] B. Fornberg, G. Wright, E. Larsson, Some observations regarding interpolants in the limit of flat radial basis functions, Comput. Math. Appl. 47 (2004) 37-55.

[12] B. Fornberg, J. Zuev, The runge phenomenon and spatially variable shape parameters in rbf interpolation, ComputMathAppl 54 (2007) 379-398.

[13] E. Fuselier, Sobolev-type approximation rates for divergence-free and curl-free RBF interpolants, Math. Comput. 77 (2008) 1407-1423.

[14] E. Fuselier, F. Narcowich, J. Ward, G. Wright, Error and stability estimates for surface-divergence free RBF interpolants on the sphere, Math. Comput. 78 (2009) 2157-2186.

[15] E.J. Fuselier, Improved stability estimates and a characterization of the native space for matrixvalued RBFs, Adv. Comput. Math. 29 (2008) 269-290.

[16] J. Gerbeau, C. Le Bris, M. Bercovier, Spurious velocities in the steady flow of an incompressible fluid subjected to external forces, Int. J. Meth. Fluids 25 (1997) 679-695.

[17] D. Handscomb, Interpolation and differentiation of multivariate functions and interpolation of divergence-free vector fields using surface splines, Technical Report 91/5, Oxford University, Numerical Analysis Group, 1991.

[18] D. Handscomb, Local recovery of a solenoidal vector field by an extension of the thin-plate spline technique, Numer. Algorithms 5 (1993) 121-129.

[19] C. Hirsch, Numerical computation of internal and external flows: the fundamentals of computational fluid dynamics, volume 2, Butterworth-Heinemann, 2007.

[20] D. Kosloff, H. Tal-Ezer, A modified Chebyshev pseudospectral method with an $\mathcal{O}\left(N^{-1}\right)$ time step restriction, J. Comput. Phys. 104 (1993) 457-469.

[21] E. Larsson, B. Fornberg, Theoretical and computational aspects of multivariate interpolation with increasingly flat radial basis functions, Comput. Math. Appl. 49 (2005) 103-130.

[22] Y.J. Lee, G.J. Yoon, J. Yoon, Convergence of increasingly flat radial basis interpolants to polynomial interpolants, SIAM J. Math. Analysis 39 (2007) 537-553.

[23] S. Lowitzsch, Error estimates for matrix-valued radial basis function interpolation, J. of Approx. Theory 137 (2005) 238-249.

[24] S. Lowitzsch, Matrix-valued radial basis functions: stability estimates and applications, Adv. Comput. Math. 23 (2005) 299-315.

[25] A.A. Mitrano, divfree-src, 2015. URL: http://dx.doi.org/10.5281/zenodo.15647, doi:10.5281/ zenodo.15647.

[26] F.J. Narcowich, J.D. Ward, Generalized Hermite interpolation via matrix-valued conditionally positive definite functions, Math. Comput. 63 (1994) 661-687. 
[27] F.J. Narcowich, J.D. Ward, G.B. Wright, Divergence-free RBFs on surfaces, J. of Fourier Analysis Appl. 13 (2007) 643-663.

[28] R.B. Platte, How fast do radial basis function interpolants of analytic functions converge?, IMA J. Appl. Math. 31 (2011) 1578-1597.

[29] R.B. Platte, T.A. Driscoll, Polynomials and potential theory for Gaussian radial basis function interpolation, SIAM J. Numer. Analysis 43 (2005) 750-766.

[30] R.B. Platte, L.N. Trefethen, Chebfun: A new kind of numerical computing, in: Progress in Industrial Mathematics at ECMI 2008, Springer, 2010, pp. 69-87.

[31] R.B. Platte, L.N. Trefethen, A.B. Kuijlaars, Impossibility of fast stable approximation of analytic functions from equispaced samples, SIAM Rev. 53 (2011) 308-318.

[32] R. Schaback, Multivariate interpolation by polynomials and radial basis functions, Constr. Approx. 21 (2005) 293-317.

[33] A. Tolstykh, D. Shirobokov, On using radial basis functions in a "finite difference mode" with applications to elasticity problems, Comput. Mech. 33 (2003) 68-79.

[34] A. Townsend, L.N. Trefethen, An extension of Chebfun to two dimensions, SIAM J. Sci. Comput. 35 (2013) C495-C518.

[35] H. Wendland, Scattered data approximation, volume 17, Cambridge University Press, 2005.

[36] G.B. Wright, B. Fornberg, Scattered node compact finite difference-type formulas generated from radial basis functions, J. Comput. Phys. 212 (2006) 99-123. 